\title{
Nonmigrating tidal signatures in the magnitude and the inter-hemispheric asymmetry of the equatorial ionization anomaly
}

\author{
C. Xiong ${ }^{1,2}$ and H. Lühr ${ }^{1}$ \\ ${ }^{1}$ Helmholtz Centre Potsdam, GFZ German Research Centre for Geosciences, Telegrafenberg, 14473 Potsdam, Germany \\ ${ }^{2}$ Department of Space Physics, College of Electronic Information, Wuhan University, 430079 Wuhan, China
}

Correspondence to: C. Xiong (xiongchao@whu.edu.cn)

Received: 21 February 2013 - Revised: 22 May 2013 - Accepted: 23 May 2013 - Published: 27 June 2013

\begin{abstract}
Based on nine years of observations from the satellites CHAMP and GRACE the tidal signatures in the magnitude and the inter-hemisphere asymmetry of the equatorial ionization anomaly (EIA) have been investigated in this study. The EIA magnitude parameters show longitudinal wavenumber 4 and 3 (WN4/WN3) patterns during the months around August and December, respectively, while for different EIA parameters the contributions of the various tidal parameters are different. For the crest-to-trough ratio (CTR) the dominating nonmigrating tidal component contributing to WN4 is DE3 during the months around August, while during the months around December solstice the stationary planetary wave, SPW3, takes a comparable role to DE2 in contributing to WN3. For the apex height index (ApexHC) of the EIA fluxtube the stationary planetary waves, SPW4/SPW3, exceed the amplitudes of DE3/DE2 taking the leading role in causing the longitudinal WN4/WN3 patterns. During the months around December solstice the SW3 tide is prominent in both CTR and ApexHC. SW3 shows a strong dependence on the solar flux level, while it is hardly dependent on magnetic activity. For the EIA interhemispheric asymmetry only WN1 and WN2 longitudinal patterns can be seen. During June solstice months the pattern can be explained by stationary planetary waves SPW1 and SPW2. Conversely, around December solstice months longitudinal features exhibit some local time evolution, in particular the diurnal nonmigrating tide D0 takes the leading role.
\end{abstract}

Keywords. Ionosphere (equatorial ionosphere; ionosphereatmosphere interactions) - Meteorology and atmospheric dynamics (waves and tides)

\section{Introduction}

In recent years growing evidence is provided about longitudinal modulation of ionospheric quantities by tidal effects originating from the tropical troposphere. These atmospheric tides excited in the troposphere by latent heat release in deep convective tropical clouds can vertically propagate and achieve wind amplitudes in the order of tens of $\mathrm{ms}^{-1}$ in the mesosphere-lower thermosphere (MLT) region (Hagan and Forbes, 2002). In the ionospheric $\mathrm{E}$ region above $90 \mathrm{~km}$ the tides interact with charged particles. There they modulate the background zonal electric field and daytime $\boldsymbol{E} \times \boldsymbol{B}$ vertical plasma drift in the equatorial region. The electric fields map into the equatorial $\mathrm{F}$ region along magnetic field lines, causing longitudinal wave structures in the $\mathrm{F}$ region plasma density, as observed from a quasi-sun-synchronous platform in space (Forbes et al., 2008). Using $1356 \mathrm{~nm}$ airglow brightness measurements, Sagawa et al. (2005) first reported about a four-peaked longitudinal pattern, the wavenumber-4 (WN4) structure of the equatorial ionization anomaly (EIA). After that the WN4 structure was found in satellite observations of several ionospheric parameters, such as total electron content (TEC), vertical plasma drift and the equatorial electrojet (EEJ) (Scherliess et al., 2008; Kil et al., 2007; England et al., 2006; Lühr et al., 2008). Immel et al. (2006) and Wan et al. (2010) suggested that the WN4 structure corresponds well to the diurnal DE3 nonmigrating tidal component. Based on multi-years CHAMP observations, Lühr et al. (2008) revealed that during solstice months there appears a prominent wavenumber-3 (WN3) longitudinal pattern in EEJ intensity. In their consecutive study they presented a comprehensive decomposition of tidal signatures of the three important ionospheric quantities, EEJ, vertical plasma 
drift and crest-to-trough ratio (CTR) of the EIA (Lühr et al., 2012). Their results demonstrate that DE3 is dominating the tidal spectrum during the months around August in the ionospheric quantities, and disappears around December solstice; while the DE2 tide peaks during solstice months and shows minima around equinoxes.

It is well accepted that latent heat released from deep convection in the tropical troposphere is the major source of the nonmigrating tides DE2 and DE3 (Hagan and Forbes, 2002, 2003). These authors state that DE3 tides dominate the temperature and zonal wind response at low latitude near $100 \mathrm{~km}$ altitude during most of the year. In tidal terminology the first letter, D, stands for diurnal, $\mathrm{S}$ for semidiurnal and the second letter, E, for eastward and $\mathrm{W}$ for westward propagation. The number at the end represents the zonal wavenumber, i.e., number of wave maxima that exists simultaneously around the globe. The DE2 and DE3 tidal components cause prominent modulations of the zonal winds, which obtain largest amplitudes around $105 \mathrm{~km}$ altitude (e.g., Oberheide et al., 2009), and these winds can generate currents in the ionospheric $\mathrm{E}$ layer. These tides are often assumed to be the leading contributor to the WN4/WN3 longitudinal structures in the ionosphere. In a numerical simulation study Hagan et al. (2009) revealed that the nonlinear interaction between the migrating diurnal tide DW1 and nonmigrating DE3 can lead to a stationary planetary wave 4 (SPW4) and a semidiurnal SE2 tide in the equatorial zonal wind at $\mathrm{E}$ region heights. The combined amplitudes of these two resulting components can be comparable with that of the DE3 (Oberheide et al., 2011). Furthermore, based on observations from the Constellation Observing System for Meteorology, Ionosphere, and Climate (COSMIC) satellites and on simulations with the Ground-to-topside model of Atmosphere and Ionosphere for Aeronomy (GAIA), Pancheva and Mukhtarov (2011), Pancheva et al. (2012) showed the important contribution of the stationary planetary waves, SWP4/SWP3, to the WN4/WN3 ionospheric structures as well as the dependence of the WN4/WN3 hemisphere asymmetry on the SE2/SE1 tides.

In the study of Lühr et al. (2012) (comparing Figs. 5 and 7), a remarkable one-to-one agreement between the seasonal variations of DE2 and DE3 tidal components in the EEJ and the E layer zonal wind can be seen, as well as of the ratio between the two tidal components. Since the electrojet is related to the vertical electric field, this convincing match is a strong argument for a direct modulation of the ambient electric field caused by the zonal wind. Further, Lühr et al. (2012) analysed the annual variations of all components in the tidal spectra separately for the EEJ, vertical plasma drift and CTR. Besides DE2 and DE3, which are excited by deep tropical convection in the troposphere and exhibiting a distinctly different seasonal variation, WN1 and WN2 seem to be generated primarily by an interaction of the migrating tides with longitudinal ionospheric structures.
In our previous study (Xiong et al., 2013), the seasonal and local time variations of the EIA magnitude as well as interhemisphere asymmetry were investigated. In this consecutive study, we will mainly focus on the longitudinal patterns of the EIA. As is well known, the EIA is mainly formed by the equatorial fountain effect via upward $\boldsymbol{E} \times \boldsymbol{B}$ plasma drift driven by a large-scale eastward electric field near the magnetic dip equator (Duncan, 1959), and the crest-to-trough latitudinal distance indicates the strength of the daytime ionospheric electric field (Rishbeth, 2000). We use in this study the magnetic latitudes of the ionization crests to characterise the EIA strength. Besides the nonmigrating tidal signatures of the EIA magnitude we present for the first time the tidal signatures of the EIA inter-hemispheric asymmetry parameters. Nearly nine years of common electron density observations from CHAMP and GRACE have provided a good opportunity for such a research spanning a wide range of different solar activity levels.

In Sect. 2 we first introduce a set of parameters to describe the magnitude and the inter-hemispheric asymmetry of the EIA. Then we focus on the longitudinal WN4 and WN3 patterns of the EIA magnitude parameters during the months around August and around December solstice when the DE3 and DE2 tidal components are known to be largest, respectively. Further, we investigate the prominent tidal signatures of EIA inter-hemisphere asymmetry parameters during solstice months. At last we try to derive tidal spectra by fitting synthetic signals to our observations and finally discuss the results in the context of previous publications.

\section{Datasets and processing approach}

\subsection{Datasets}

The CHAMP satellite was launched on 15 July 2000 into a circular, near-polar orbit (inclination: $87.3^{\circ}$ ) with on initial altitude of $456 \mathrm{~km}$. By the end of 2009 the orbit has decayed to $310 \mathrm{~km}$. The local time of the orbital plane changes by $1 \mathrm{~h}$ in 11 day, requiring about 130 days for covering all local times (Reigber et al., 2002). The Planar Langmuir Probe (PLP) on board the satellite was taking measurements of the electron density and temperature every $15 \mathrm{~s}$. The PLP electron density readings have been validated by comparison against digisonde measurements at Jicamarca (McNamara et al., 2007).

GRACE, comprising two spacecraft GRACE-A and GRACE-B, was launched on 17 March 2002 into a nearcircular, polar orbit (inclination: $89^{\circ}$ ) with an initial altitude of about $490 \mathrm{~km}$. The altitude of the two spacecraft is quite stable over the years, which stays around $480 \mathrm{~km}$. The local time of the orbital plane precesses by 4.5 min every day taking the mission 160.5 days to sample all local times (Tapley et al., 2004). The two spacecrafts follow each other at a distance of about $170 \sim 220 \mathrm{~km}$. The total electron content 
(TEC) between the spacecraft can be deduced from the KBand Ranging (KBR) data. When dividing the horizontal TEC by the distance between the spacecraft, the averaged electron density can be derived. A more detailed description of the GRACE electron density retrieval is given in Sect. 3 of Xiong et al. (2010) and Lühr and Xiong (2010).

\subsection{EIA description parameters}

Since CHAMP and GRACE are both near circular, polar orbiting satellites, the electron density at two almost constant altitudes are recorded. These latitudinal electron density profiles at constant altitudes are used to map the EIA. In this study, we have developed an automatic subroutine to find the EIA crests and trough only if the two crests are formed. For more details of the selection procedure see Xiong et al. (2013). After the automatic selection we have checked all the events individually to make sure that they are good for our EIA study. The observations from CHAMP (January 2001 to December 2009) and GRACE (April 2002 to October 2009) are considered for this study only from quiet magnetic conditions $(\mathrm{Kp} \leq 3.5)$.

On each satellite orbit we record the electron densities and magnetic latitudes of the EIA crests in the two hemispheres and the trough as $N_{\mathrm{e}_{\text {south_c }}}, N_{\mathrm{e}_{\text {north_c }}}, N_{\mathrm{e}_{\text {trough }}}, \beta_{\text {south_c }}$, $\beta_{\text {north_c }}$, and $\beta_{\text {trough }}$, respectively. Then we introduce the CTR, the Mean Crest latitude (MCL) and Apex Height of Crest (ApexHC) for representing the peak latitude of the EIA. They are calculated as outlined below:

$\mathrm{CTR}=\frac{N_{\mathrm{e}_{\text {north_c }}}+N_{\mathrm{e}_{\mathrm{e}_{\text {outh_c }}}}}{2 \cdot N_{\mathrm{e}_{\text {trough }}}}$
$\mathrm{MCL}=\frac{\beta_{\text {north_c }}-\beta_{\text {south_c }}}{2}$

ApexHC $=\frac{1}{2} \cdot\left(\right.$ ApexHC $_{\text {north_c }}+$ ApexHC $\left._{\text {south_c }}\right)$

$=\frac{R_{\mathrm{E}}}{2} \cdot\left\{\left(\frac{r_{\text {north_c }}}{R_{\mathrm{E}}} \cdot \frac{1}{\cos ^{2} \beta_{\text {north_c }}}-1\right)+\left(\frac{r_{\text {south_c }}}{R_{\mathrm{E}}} \cdot \frac{1}{\cos ^{2} \beta_{\text {south_c }}}-1\right)\right\}$

where $r_{\text {north_c }}, r_{\text {north_c }}$ are the radial distances of the measurement points from the Earth's centre, $R_{\mathrm{E}}=6378 \mathrm{~km}$ is the Earth's radius near the equator. Under the assumption that the equatorial anomaly is aligned with magnetic fluxtubes, ApexHC is independent of the sampling height. Therefore, results from different altitudes, e.g., the beginning and end of the CHAMP mission or CHAMP and GRACE comparisons, are easier to interpret than the variations of MCL. We regard the apex height of the crests as an indirect measure of the fountain effect strength.

The Asymmetry of Crest Latitude (ACL) is defined as:

$\mathrm{ACL}=\beta_{\text {north_C }}+\beta_{\text {south_C }}$

Processes in addition to the fountain effect are required to facilitate the latitudinal displacement to the EIA.

\subsection{Processing approach}

Harmonic longitudinal structures observed by near-polar orbiting satellites can be caused by a multitude of tidal components. A general mathematical formulation of the relation between longitudinal patterns in satellite observations and the nonmigrating tidal description in the Earth-fixed frame is given by Forbes et al. (2006) or by Häusler and Lühr (2009) in their Sect. 2. For the actual determination of the tidal signatures, we first sort the EIA parameters into local time $(1 \mathrm{~h})$ and longitude $\left(15^{\circ}\right)$ bins, then the longitudinal mean values determined separately for all local time hours are subtracted from the measurements. By removing the longitudinal mean values hour by hour the dominating diurnal migrating tide is suppressed and the nonmigrating tides appear clearer. These mean-free data are further processed by the one-dimensional Fourier transform which will bring forth the sum of observed tidal signatures for each wavenumber. From the longitude variation with local time, we will determine the dominant tidal components for each wavenumber and use this set of selected tides for reproducing the observations. The longitudinally averaged local time variations of the EIA parameters have earlier been studied by Xiong et al. (2013). They represent the effect of migrating tides.

\section{Results}

\subsection{Tidal signatures in the EIA magnitude parameters}

Longitudinal variations of the EIA can be related to nonmigrating tidal signals. In different seasons the most prominent tidal components have different wavenumbers. For example, the WN4, in particular the DE3 tide, is dominating the crestto-trough ratio around August and disappears around December solstice, while the WN3 is strong during solstice months and shows minima around equinoxes (Lühr et al., 2012). Following this instruction, we have selected the two contrasting intervals of a year to investigate the longitudinal structure of the EIA. These are the 130 days around 15 August and the 130 days around 1 January. They are expected to reveal quite different tidal signatures. Firstly, we looked into the variations of EIA magnitude parameters. Figure 1 presents the local time versus longitudinal structure of the MCL from the months around August for days of year (DOY) from 162 to 292. Blank areas denote a lack of observations in a bin. We can clearly see a longitudinal WN4 structure during the time sector 10:00-18:00 LT both for the high and low solar activity years. In CHAMP and GRACE observations the wave centers appear at $-180^{\circ} \mathrm{E},-90^{\circ} \mathrm{E}, 0^{\circ} \mathrm{E}$ and $90^{\circ} \mathrm{E}$, among which the wave peak around $90^{\circ} \mathrm{E}$ is the strongest. Comparing the two satellite observations for the different solar activity years, the WN4 structure of MCL is less obvious in GRACE observations during lower solar activity years (right bottom panel). It may be that at local times several hours 

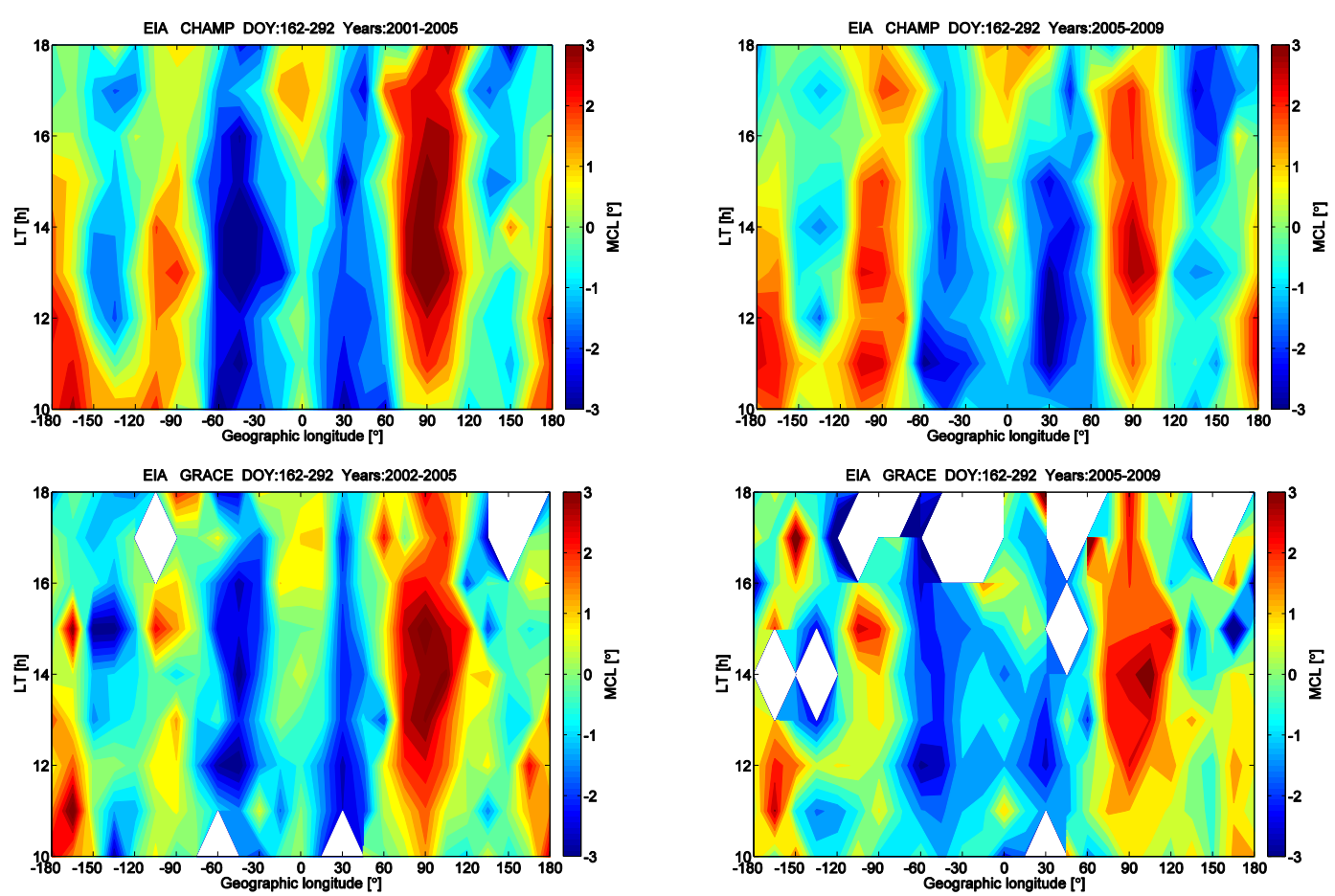

Fig. 1. The local time versus longitudinal distribution of the EIA latitude parameter MCL from CHAMP (top) and GRACE (bottom) observations during higher (left) and lower (right) solar activity years around August; data are from DOY between 162 and 292.

apart from noon the EIA does not reach the GRACE altitudes during low solar activity years. When looking at the local time variation of the pattern in MCL, it seems there is no obvious shift in longitude with local time of the WN4 crests.

As we mentioned above, the altitudes of CHAMP and GRACE orbits decayed at different rates over time, but the ApexHC is independent of the sampling height, so we have presented in Fig. 2 also the local time versus longitudinal distribution of ApexHC around August. Clearer and smoother WN4 structures can be seen within the local time sector 10:00-18:00 LT from both satellite observations. The EIA fountain effect reaches highest around 14:00 LT near the longitude of $90^{\circ} \mathrm{E}$. Also for ApexHC, the local time (LT) evolution of WN4 is not as expected for DE3. Conversely, the DE3 in CHAMP data still takes the leading role in CTR among all the tidal components during the months around August, as presented by Lühr et al. (2012). Considering our observations as shown here in Figs. 1 and 2, it seems CTR and ApexHC are affected by different coupling mechanism. In the discussion part we will come back to the comparison between CTR and ApexHC.

Because the ApexHC is independent of the sampling height and thus preferable, we do not show any further results of the latitude parameter MCL in the rest of our study. Figure 3 presents the local time versus longitudinal distribution of ApexHC for months around December solstice from DOY between 300 and 65 . Wave centers appear at longitudes around $-165^{\circ} \mathrm{E},-30^{\circ} \mathrm{E}$, and $90^{\circ} \mathrm{E}$. As for the local time variation, a slightly eastward tilted $\mathrm{WN} 3$ structure can be seen. Comparing the WN3 with the WN4 structure in Fig. 2, it seems that WN3 is more scattered during December solstice months, especially at GRACE altitude during low solar activity years. Obviously, different tidal components are interfering.

\subsection{Tidal signatures of the EIA inter-hemispheric asym- metry parameters}

In this section, we have a look at the local time versus longitudinal distribution of the EIA inter-hemispheric asymmetry parameter, ACL. At first we focused on the months around August and then around December solstice. In both cases neither WN4 nor WN3 structures dominate. Then we made a survey through the observations month-by-month. Some interesting features showed up, especially during solstice months. Figure 4 presents the local time versus longitudinal distribution of the ACL for the 130 days around June solstice during high (left) and low (right) solar activity years both from CHAMP (top) and GRACE (bottom) observations. At CHAMP altitude, the EIA is displaced significantly northward into the summer hemisphere in the longitude sector between $-180^{\circ} \mathrm{E}$ and $-75^{\circ} \mathrm{E}$. Conversely, in the remaining longitudinal sector, $-30^{\circ} \mathrm{E}$ and $120^{\circ} \mathrm{E}$, the EIA is slightly shifted into the Southern (winter) Hemisphere. At GRACE 

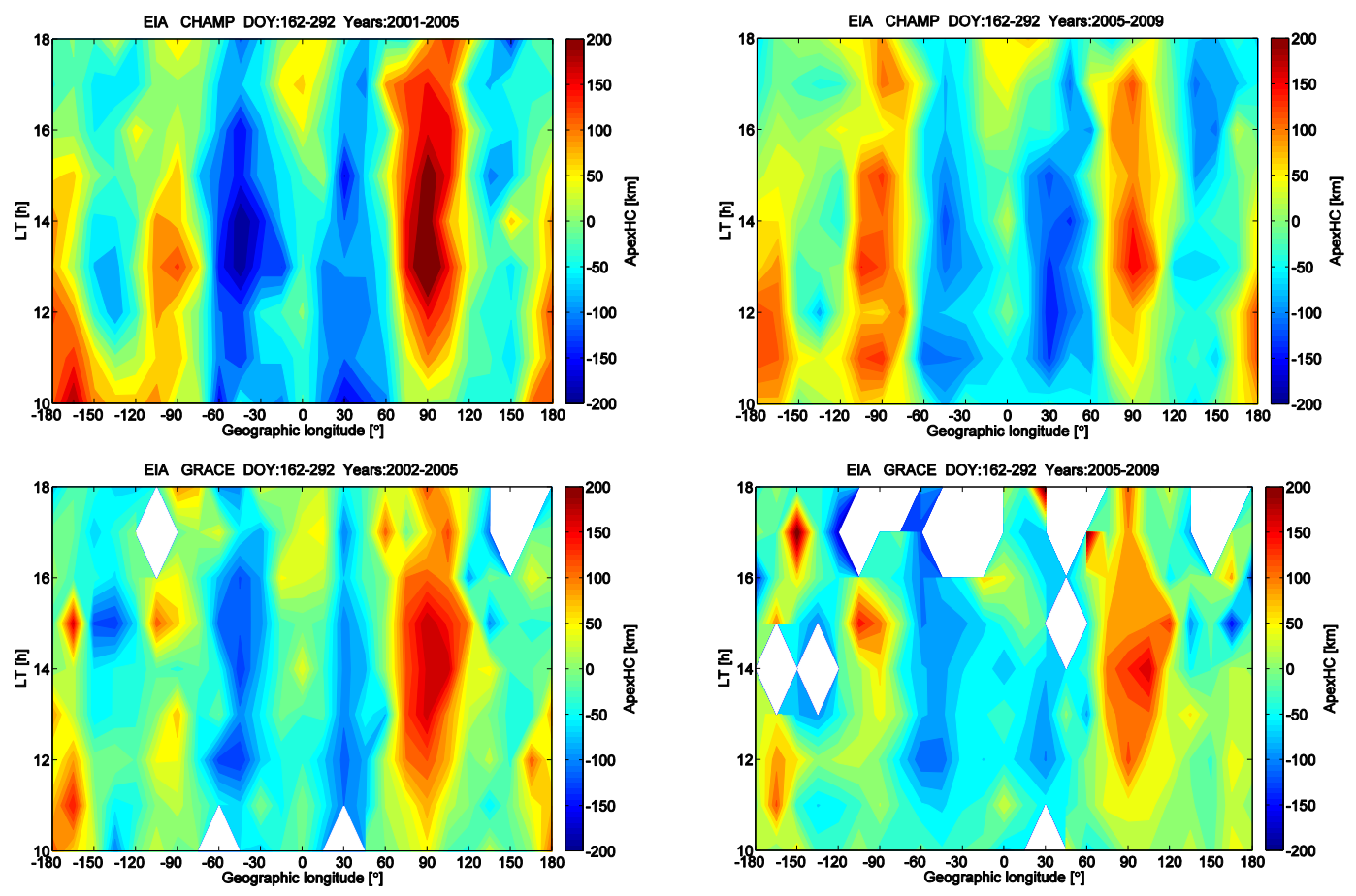

Fig. 2. Same as Fig. 1, but for the EIA parameter ApexHC.
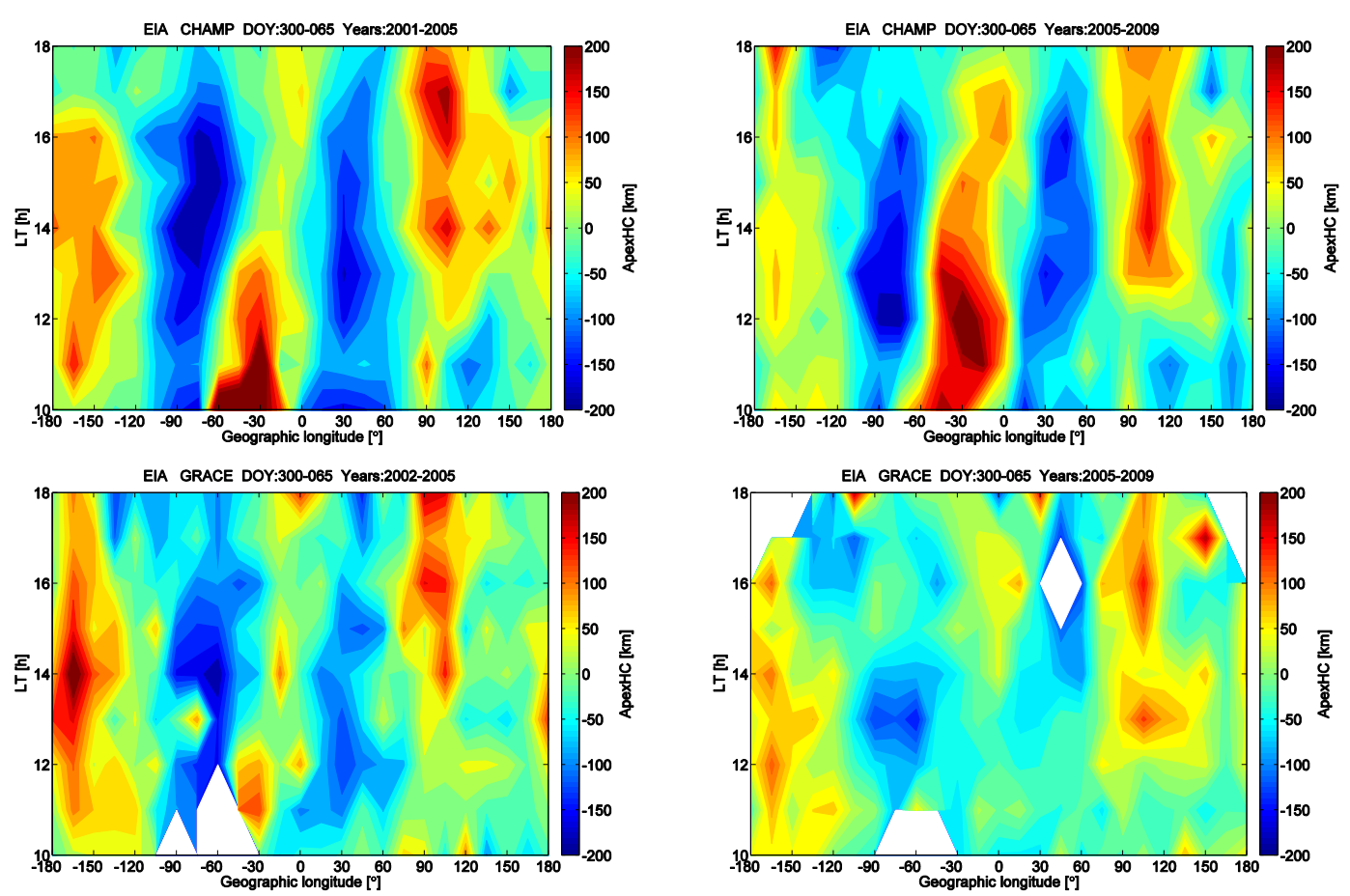

Fig. 3. The local time versus longitudinal distribution of the EIA parameter ApexHC from CHAMP (top) and GRACE (bottom) observations during higher (left) and lower (right) solar activity years around December; data are from DOY between 300 and 065.

altitude the EIA shows similar latitudinal asymmetries between the two hemispheres but at slightly lower values.
Similarly, Fig. 5 shows the same local time versus longitudinal distribution of the ACL, but for the 130 days around December solstice. When comparing to June solstice months, 

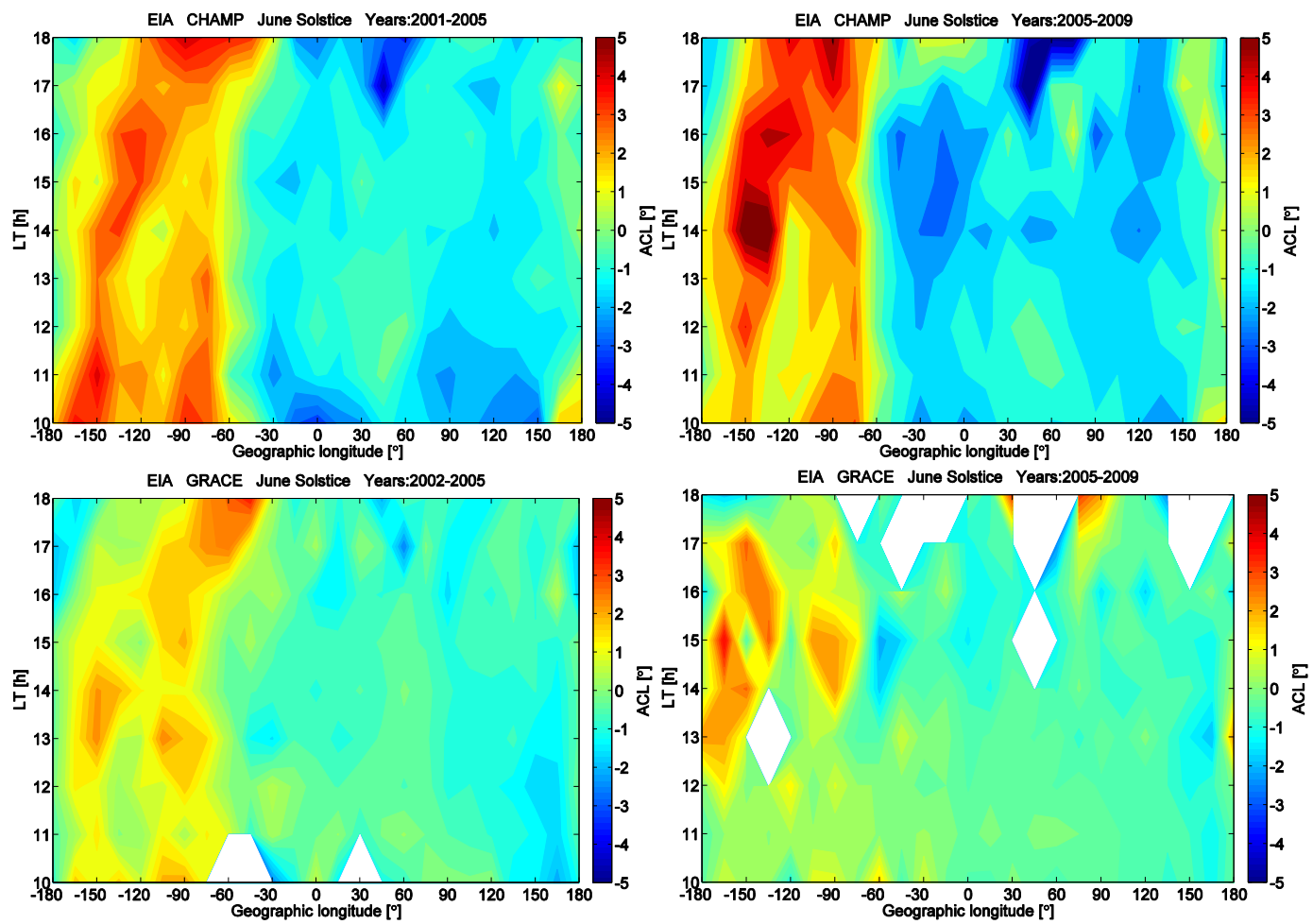

Fig. 4. The local time versus longitudinal distribution of the EIA inter-hemispheric asymmetry parameter ACL around June solstice months from CHAMP (top) and GRACE (bottom) observations during higher (left) and lower (right) solar activity years.
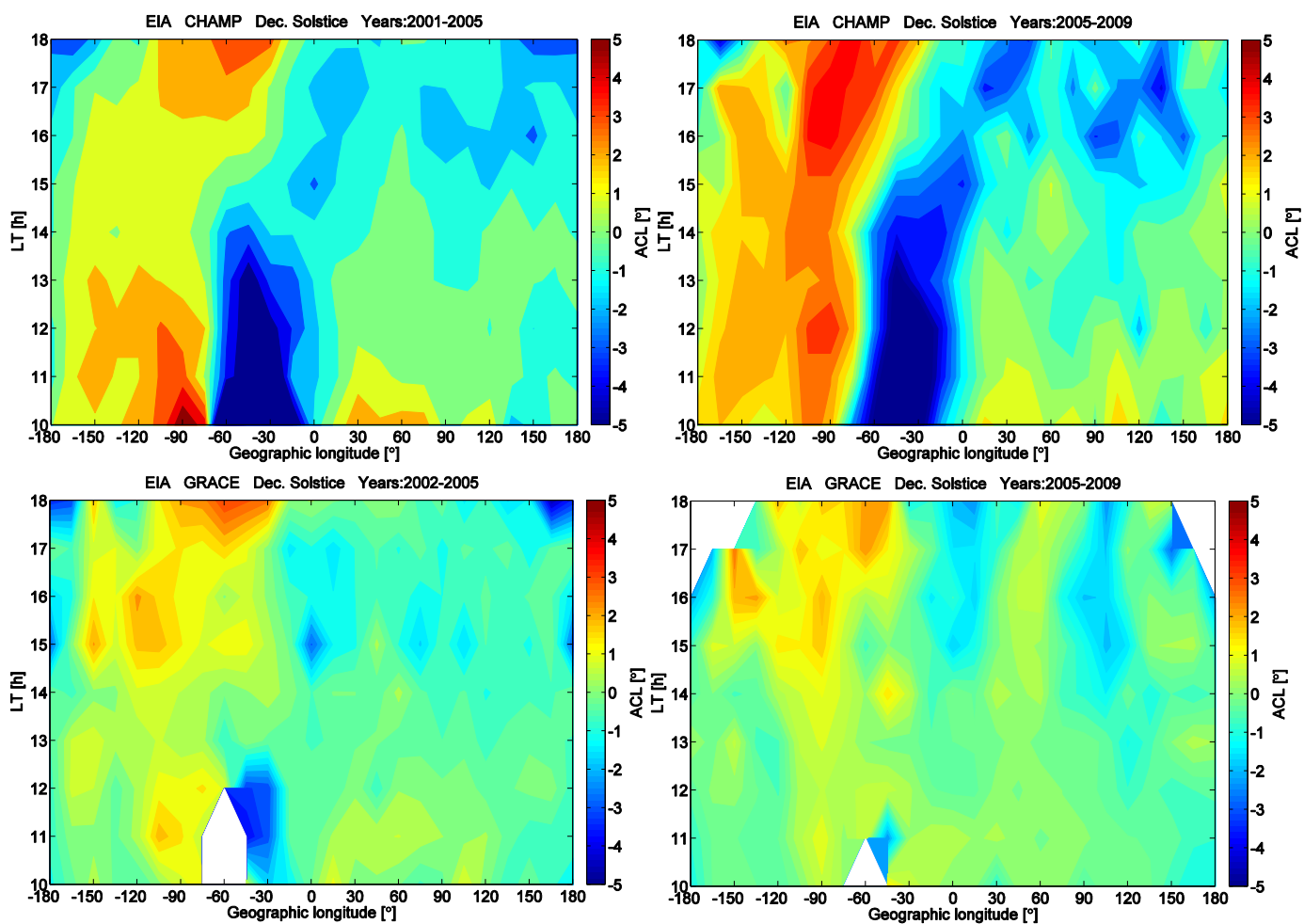

Fig. 5. Same as Fig. 4, but for December solstice months. 

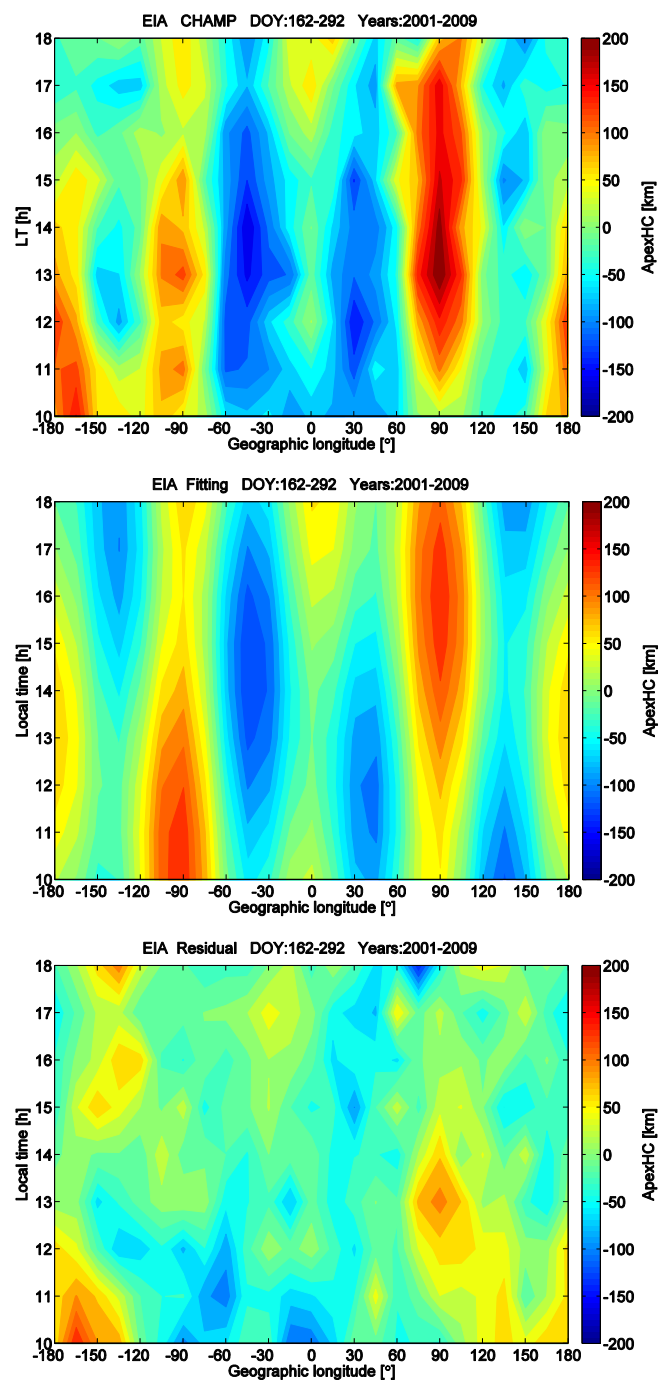
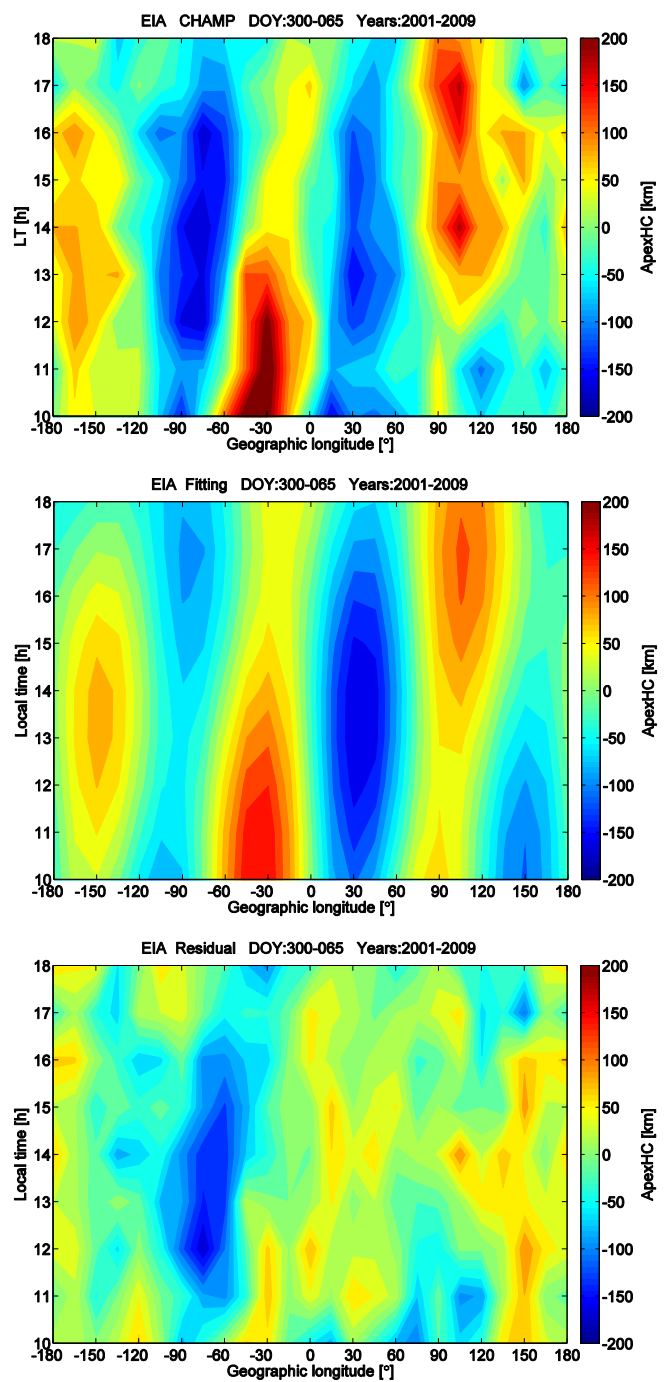

Fig. 6. The local time versus longitudinal distribution of ApexHC, observations (top), synthetic signals (middle) and residual (bottom) during the months around August (left) and December solstice (right).

between longitudes of $-180^{\circ} \mathrm{E}$ and $-75^{\circ} \mathrm{E}$, larger ACL values can be also seen here at both satellite altitudes. Different from that, positive ACL values appear also between $0^{\circ} \mathrm{E}$ and $90^{\circ} \mathrm{E}$, which are most clear during the hours 10:00 to 12:00 LT at CHAMP altitude. At GRACE altitude the positive features extend to later local time, especially during lower solar activity years. The minimum of ACL around $-30^{\circ} \mathrm{E}$ is more prominent around December than during June solstice months. Furthermore, all the longitudinal structures show a slight eastward tilt, opposed to the patterns around June.

\section{Interpretation of the tidal signatures}

As mentioned above, the longitudinal structures observed by near-polar orbiting satellites can be caused by a multitude of tidal components. In the local time frame, in general, the tidal signatures can be expressed as:

$A_{n, s} \cos \left(n \Omega t_{\mathrm{LT}}+(s-n) \lambda-\varphi_{n, s}\right)$

where, $A_{n, s}$ is the amplitude of a tide, $n$ denotes the harmonics of a solar day, $s$ is the zonal wavenumber, $\Omega$ the rotation rate of the Earth, $t_{\mathrm{LT}}$ the local time, $\lambda$ the longitude, and $\varphi_{n, s}$ the phase of the tide (Forbes et al., 2006; Häusler and Lühr, 2009). We can see that the migrating tides $(s-n=0)$ are independent of longitude in the local time frame, while the nonmigrating tides $(s-n \neq 0)$, will show longitudinal patterns at different wavenumbers. Taking WN4 as an example, the satellite observations cannot be related unambiguously to a certain tidal component since it can be caused by any tide for which holds $|s-n|=4$. In other words, the observed WN4 pattern can be caused by a diurnal tide $(n=1)$ with $s=-3$ or $s=+5$, a semidiurnal tide $(n=2)$ with $s=-2$ or 

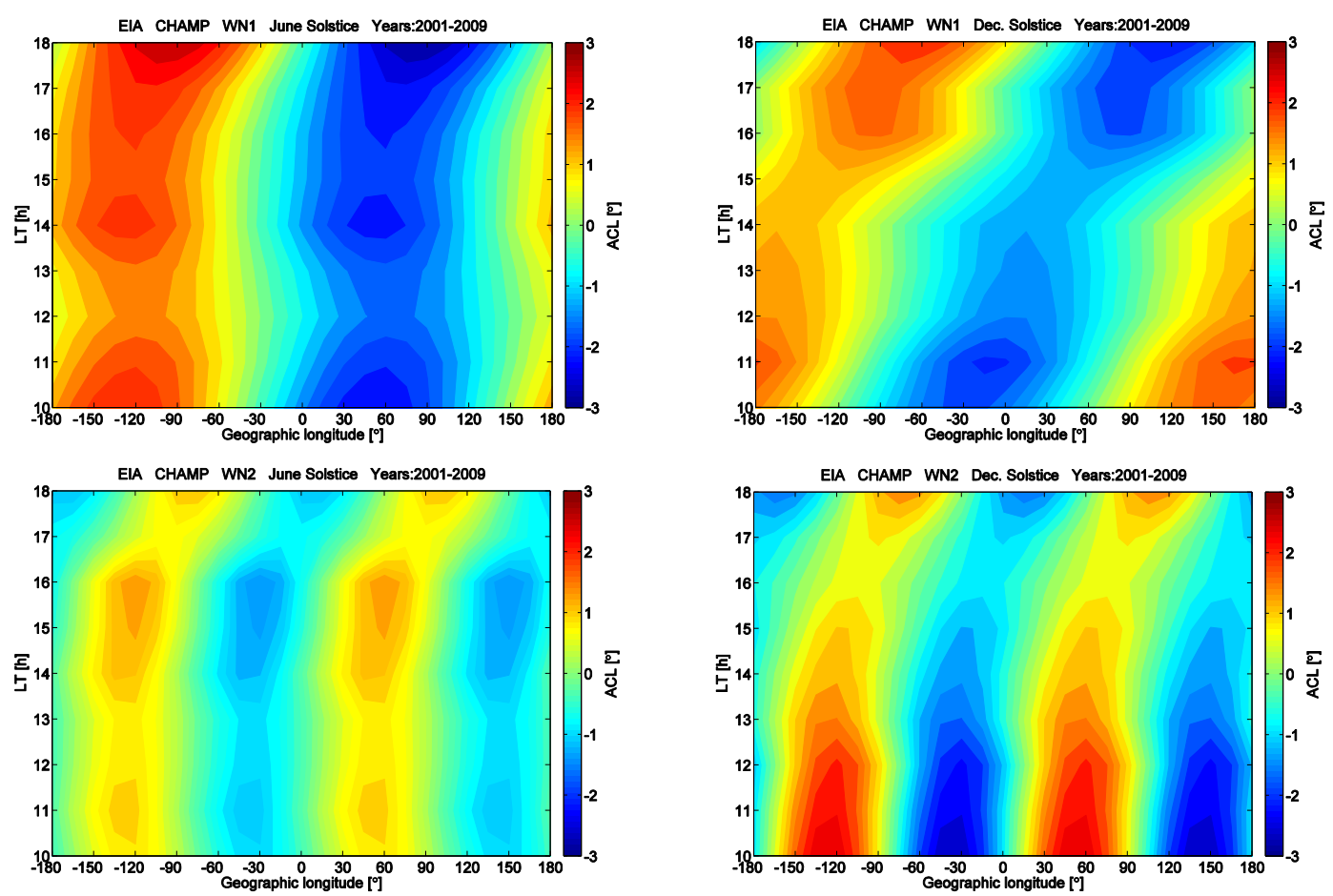

Fig. 7. Filtered data of EIA inter-hemispheric asymmetry from the months around June (left) and December (right), zonal wavenumber 1 (top) and wavenumber 2 (bottom).

$s=+6$, or by a stationary planetary wave $(n=0)$ with $s=4$. In order to quantify the responsible oscillations for the WN4 structure, we have to identify the individual contributions. This can be done by looking at the tilt angle of the observed WN4 structure.

\subsection{The tidal signature of the EIA apex height}

For this analysis we combine the data from 2001 to 2009. At first, we apply separately for each local time bin a Fourier transform to the ApexHC data from CHAMP and GRACE observations along longitude. Spectra for wavenumbers 1 through 4 of ApexHC have been analysed separately for the months around August and around December solstice. By comparing the amplitudes and assessing the phase behaviour, the tidal components of SPW4, SPW2, DE3 and SW3 are found to be most prominent during the months around August. Conversely, the SPW3, SPW2, DE2 and SW3 are dominating during the months around December solstice. Then we adjust the four most prominent tidal components for the two time intervals, respectively, to reconstruct tidal spectra of the ApexHC which fit best the observations.

Figure 6 presents the ApexHC observations (top), reconstructed synthetic signals (middle) and residuals (bottom) for the months around August (left) and December solstice (right). The residuals are defined as the observations minus the synthetic signal. For both the longitudinal WN4/WN3 structures, the residuals are quite randomly scattered, imply- ing that the fitting is sufficiently consistent with the observations. The amplitudes and phases of the synthetic tidal components are list in Table 1. In addition DW1 has been listed for later reference. As expected, not the DE3/DE2, but the stationary planetary waves, SPW4/SPW3 are the dominating tidal components for the longitudinal WN4/WN3 structures of ApexHC, with amplitudes of about $57 / 50 \mathrm{~km}$ in altitude. This is in line with the LT evolution in the ApexHC which is quite stationary. A slight eastward LT evolution can be seen in ApexHC during the months around December solstice, hence DE2 is found to be the secondary tidal component with an amplitude of about $43 \mathrm{~km}$ in altitude, while the DE3 is really weak during the months around August, which is unexpected. During the months around August larger value of ApexHC at $-90^{\circ}$ and $90^{\circ} \mathrm{E}$ imply a WN2 structure corresponding to SPW2. Furthermore, the similar amplitude of SPW2 during both time intervals may imply the same exciting source. Besides the stationary planetary waves, to our surprise, the SW3 tide, which contributed to a longitudinal WN1 structure, plays an important role for the ApexHC with amplitudes of about $41 \mathrm{~km}$ at both altitudes during the months around August and around December solstice.

\subsection{Tides of the EIA hemispheric asymmetry}

Following the analysis above, we also apply the onedimensional Fourier transform to the ACL data, to separate the different wavenumber components. As is obvious from 
Table 1. Amplitudes and phases of the most prominent tidal components contributing to the modulation of ApexHC during the months around August and December solstice.

\begin{tabular}{lccc}
\hline Time interval & Tidal component & Amplitude (km) & Phase \\
\hline \multirow{3}{*}{ Months around August } & DW1 & 73.37 & - \\
& SPW4 & 57.91 & $-1^{\circ}$ \\
& SPW2 & 37.54 & $79^{\circ}$ \\
& DE3 & 12.41 & $12.4 \mathrm{~h}$ \\
& SW3 & 41.78 & $18.1 \mathrm{~h}$ \\
\hline \multirow{2}{*}{ Months around December solstice } & DW1 & 86.75 & - \\
& SPW3 & 50.23 & $-33^{\circ}$ \\
& SPW2 & 36.11 & $-25^{\circ}$ \\
& DE2 & 43.36 & $16.3 \mathrm{~h}$ \\
& SW3 & 40.99 & $19.9 \mathrm{~h}$ \\
\hline
\end{tabular}
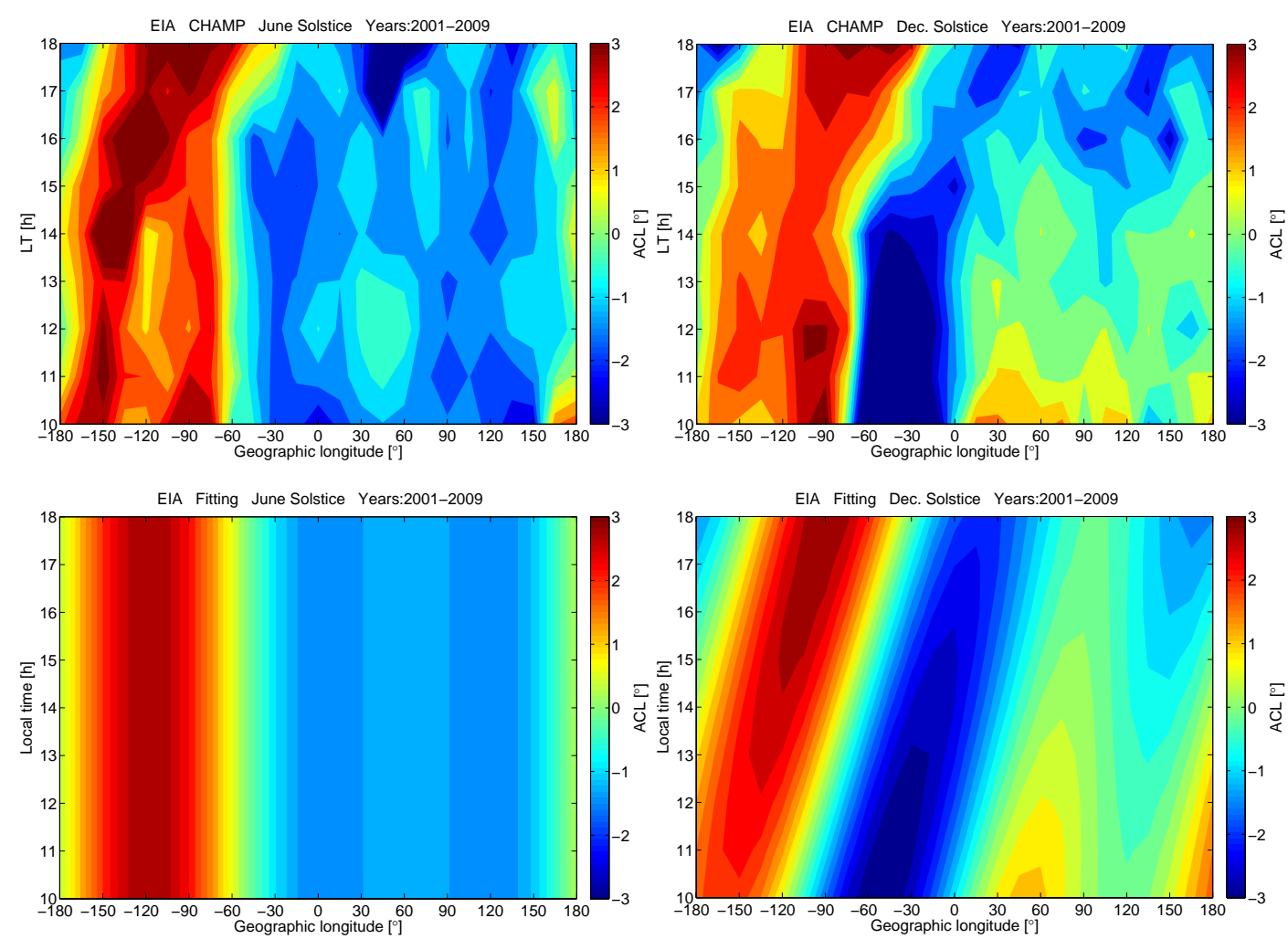

Fig. 8. The local time versus longitudinal distribution of ACL, observations (top) and synthetic signal (bottom) during the months around June (left) and December (right).

Figs. 4 and 5, neither WN4 nor WN3 patterns can be seen in the local time versus longitudinal distribution of ACL. After merging all the ACL data from 2001 to 2009, a Fourier transform has been applied separately to the June and December solstice periods. Figure 7 presents the WN1 (top) and WN2 (bottom) patterns of ACL from CHAMP observation around June (left) and December (right) solstice months. The WN1 pattern seems more stationary during June solstice and eastward titled during December solstice, while the eastward tilt of WN2 is not so prominent during December solstice.
We have applied the same fitting procedure as before. Figure 8 presents the observations (top) and synthetic signals (bottom) of CHAMP ACL both for June (left) and December (right) solstices. The amplitudes and phases of the synthetic tidal components are listed in Table 2. Besides the stationary planetary waves SPW1 and SPW2 a large amplitude of the D0 tide is needed to explain the eastward LT evolution of the WN1 structure during December solstice. The synthetic tidal signal fits the data well around June solstice, but shows some discrepancy from the observation during December months. 
Table 2. Amplitudes and phases of the most prominent tidal components contributing to the modulation of ACL during June and December solstice.

\begin{tabular}{lccc}
\hline Time interval & Tidal component & Amplitude $\left(^{\circ}\right)$ & Phase \\
\hline \multirow{2}{*}{ June solstice } & SPW1 & 2.00 & $-117^{\circ}$ \\
& SPW2 & 0.92 & $62^{\circ}$ \\
\hline \multirow{2}{*}{ December solstice } & SPW1 & 0.46 & $40^{\circ}$ \\
& SPW2 & 1.34 & $63^{\circ}$ \\
& D0 & 1.94 & $23.4 \mathrm{~h}$ \\
\hline
\end{tabular}

The implications of these tidal features will be discussed in the next section.

\section{Discussion}

Within this section we want to discuss our findings in the context of previous results related to nonmigrating tides of the EIA. In previous studies, the electron density deduced from airglow (Sagawa et al., 2005) or from CTR (Lühr et al., 2012) are used to represent the magnitude of EIA. As the winds basically modulate the background zonal electric field in the ionospheric $\mathrm{E}$ region, which maps into the ionospheric $\mathrm{F}$ region along magnetic field lines, we have to choose the magnetic latitudes and apex height of the EIA crest fluxtube in this paper as proxy for the intensity of the fountain effect, which are assumed to be closely related to the background electric field. Furthermore, we present for the first time the tidal signatures of the inter-hemispheric asymmetry of the EIA crests.

As is accepted, the atmospheric tides have different efficiencies in producing electric fields, thus will generate different wave components in the ionosphere, depending on solar activity and season (Forbes et al., 2008). At altitudes of the ionospheric $\mathrm{E}$ and $\mathrm{F}$ regions, the main spatial ionospheric variability is usually observed in satellite data as wavenumber 3 or 4 longitudinal patterns which are mostly attributed to the tidal component DE2 and DE3, respectively. England et al. (2006) had suggested that the tidal component DE3 is modelling the electrojet strength during March equinox and Lühr et al. (2008) confirmed that the DE3 tide is a very important mode for the electrojet modulation. They also reveal that during solstice months there appears a prominent WN3 longitudinal pattern in the EEJ intensity. In their consecutive study (Lühr et al., 2012), they demonstrate that the DE3 is dominating the tidal spectrum primarily during the months around August in many ionospheric quantities, and this tidal component disappears around December solstice; while the DE2 tide maximises during solstice months and shows minima around equinoxes. In Fig. 2 (bottom panel) of Lühr et al. (2012), the most intense ionization anomaly with larger CTR is observed in the Indonesian sector and the EIA is particularly well developed during the hours past noon. This is consistent with our ApexHC observation in the same longitudinal sector, where the EIA reaches apex heights beyond $800 \mathrm{~km}$ both during high and low solar activity years.

\subsection{The local time evolution}

Although the local time versus longitudinal distribution of ApexHC from both CHAMP and GRACE observations present longitudinal $\mathrm{WN} 4 / \mathrm{WN} 3$ signatures during the months around August and December, respectively, the patterns cannot simply be explained by DE3/DE2. The inconsistency in LT evolution between WN4/WN3 and DE3/DE2 creates some questions as to their origin. In fact, similar results have already been reported by previous studies. Using Global Ultraviolet Imager (GUVI) data from 2002 to 2007, He et al. (2010) showed that the WN4 structures in $[\mathrm{O}] /\left[\mathrm{N}_{2}\right]$ follows the same seasonal pattern as the strength of the DE3 tide, but does not move eastward in LT, as commonly expected. Kil et al. (2010) also found that the WN3 LT evolution in plasma density and vertical plasma drift is different from the expectation of DE2. Using the Thermosphere Ionosphere Mesosphere Electrodynamics General Circulation Model (TIME-GCM) model, Hagan et al. (2009) found that a number of waves could contribute significantly to the amplitude of WN4 structures in the zonal wind, among which are the DE3 and SPW4. These authors deduced from numerical simulation that the planetary wave SPW4 can be generated as an interaction product between the migrating wave DW1 and nonmigrating tide DE3. The interaction is most effective where the two mother tides are large, slightly above $100 \mathrm{~km}$ altitude. In Table 1 we have listed the amplitude of the dominating DW1. Similarly, England et al. (2010) found that the WN4 structure in $[\mathrm{O}] /\left[\mathrm{N}_{2}\right]$ was the result of a combination of diurnal, semidiurnal and stationary planetary waves, which may account for the unexpected LT evolution of WN4. As stationary planetary waves are believed to be generated through nonlinear interactions of the DE3/DE2 with the migrating tide, e.g., DW1, and to vary with season in the same way as the DE3/DE2 tides, therefore, it is difficult to separate these stationary planetary waves from the DE3/DE2 tides in observations when only a limited range of LT is sampled. The distinction between the two requires careful attention (England, 2011).

For checking previous results we have also looked at the local time versus longitudinal distribution of CTR. After combining the data from the years 2001 to 2009, Fig. 9 presents the local time versus longitudinal distribution of CTR both from CHAMP (top) and GRACE (bottom) observations for the months around August (left) and December solstice (right). Longitudinal WN4 patterns with a clear eastward tilted LT evolution are observed in both satellite observations (see Fig. 9, left), while WN3 patterns with no LT evolution can be seen at CHAMP altitude (see Fig. 9, right). At GRACE altitude the WN3 pattern is really weak. After applying the same fitting procedure to the CTR, the 

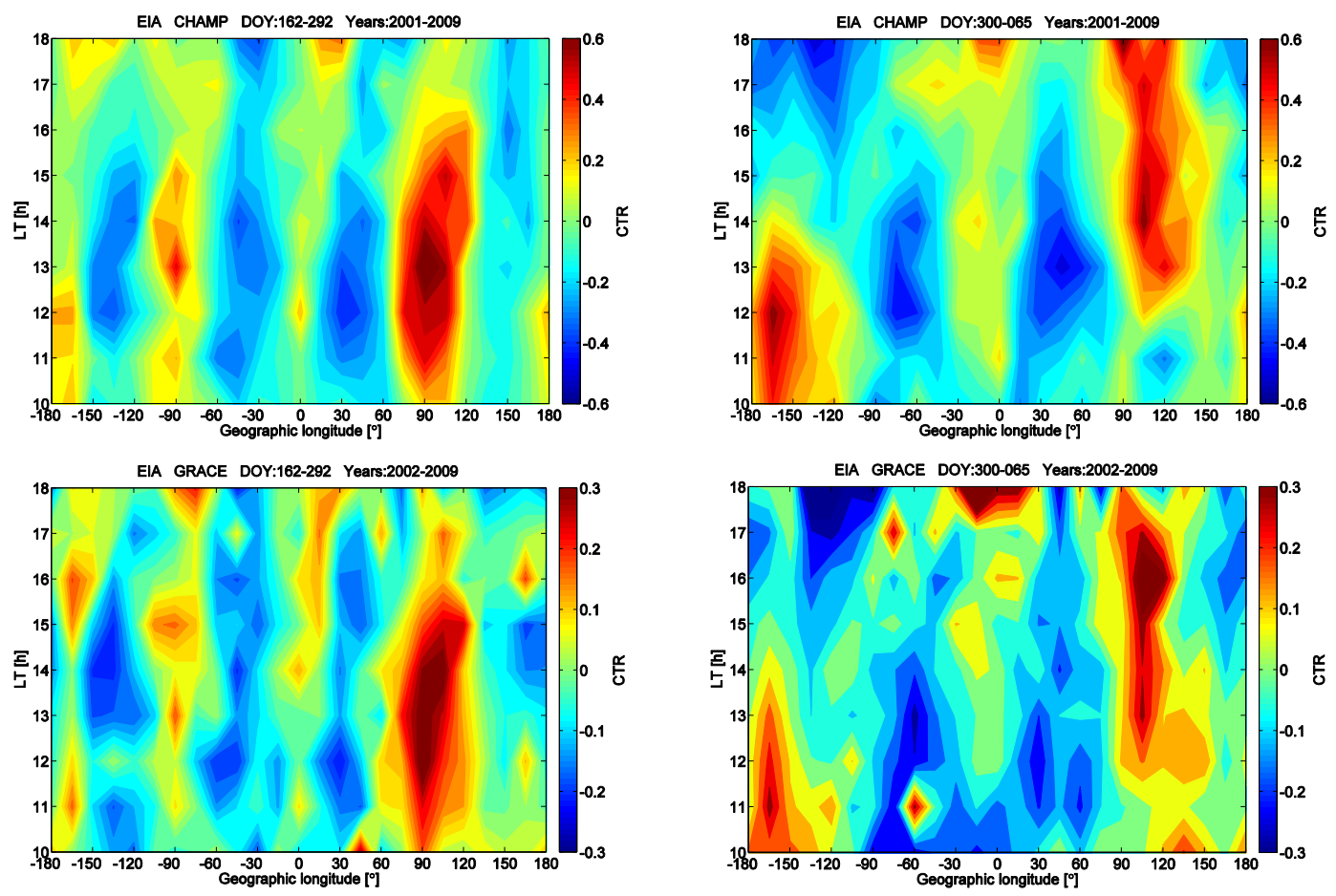

Fig. 9. The local time versus longitudinal distribution of the EIA parameter crest-to-trough ratio (CTR) from CHAMP (top) and GRACE (bottom) observations around August (left) and around December (right).

Table 3. Amplitudes and phases of the most prominent tidal components contributing to the modulation of CTR during the months around August and December solstice, from both CHAMP and GRACE observations.

\begin{tabular}{|c|c|c|c|c|c|}
\hline \multirow{2}{*}{ Time interval } & \multirow[b]{2}{*}{ Tidal component } & \multicolumn{2}{|c|}{ CHAMP } & \multicolumn{2}{|c|}{ GRACE } \\
\hline & & Amplitude & Phase & Amplitude & Phase \\
\hline \multirow{4}{*}{ Months around August } & SPW4 & 0.087 & $2^{\circ}$ & 0.043 & $11^{\circ}$ \\
\hline & SPW2 & 0.103 & $92^{\circ}$ & 0.052 & $94^{\circ}$ \\
\hline & DE3 & 0.133 & $11.5 \mathrm{~h}$ & 0.047 & $13.8 \mathrm{~h}$ \\
\hline & SW3 & 0.068 & $16.7 \mathrm{~h}$ & 0.041 & $15.8 \mathrm{~h}$ \\
\hline \multirow{4}{*}{ Months around December solstice } & SPW3 & 0.083 & $-21^{\circ}$ & 0.053 & $-13^{\circ}$ \\
\hline & SPW2 & 0.068 & $-38^{\circ}$ & 0.041 & $-37^{\circ}$ \\
\hline & DE2 & 0.076 & $16.3 \mathrm{~h}$ & 0.011 & $18.9 \mathrm{~h}$ \\
\hline & SW3 & 0.178 & $18.5 \mathrm{~h}$ & 0.090 & $17.8 \mathrm{~h}$ \\
\hline
\end{tabular}

obtained amplitudes and phases of the synthetic tidal components are list in Table 3. As expected, during the months around August DE3 is the dominating tidal component for the WN4 structure of the CTR, exceeding the contribution of SPW4, both in CHAMP and GRACE observations. During the months around December solstice, the stationary planetary wave SPW3 takes the leading role in contributing to the WN3 structure of CTR, while the DE2 is more or less weak, especially at GRACE altitude. Our observations confirm the important role of SPW4/SPW3 in contributing to the longitudinal WN4/WN3 patterns of the ionization anomaly, and it seems these stationary planetary waves are more prominent during the months around December solstice.
Conversely, the SPW4 clearly exceeds the DE3 amplitude in contributing to the longitudinal WN4 pattern of the ApexHC from CHAMP observations during the August period, as listed in Table 1. The height of the EIA fluxtube is strongly influenced by the vertical plasma velocity in the topside ionosphere. Obviously, the influence of the DE3 tide on the vertical drift is quite low at an altitude around $800 \mathrm{~km}$. Here the stationary planetary wavenumber 4 seems to control the activity. We will go into more detail in the next subsection. 


\subsection{Tides at different altitudes}

Zhang et al. (2010) using observations from Thermosphere, Ionosphere, Mesosphere Energetics and Dynamics/Global Ultraviolet Imager (TIMED/GUVI) found that throughout most of the year WN3 dominates the daytime $[\mathrm{O}] /\left[\mathrm{N}_{2}\right]$, while WN4 is only prominent during summer. They attributed this to the DE2 contribution which might be more effective in creating longitudinal patterns than that of DE3. Forbes et al. (2012) state that the tides enter the thermosphere at about $100 \mathrm{~km}$ and their spectrum evolves with height due to effects of molecular dissipation, potentially moderated by the effects of mean winds. Molecular dissipation preferentially damps the waves with shorter vertical wavelengths, allowing the longest scale waves to reach, e.g., CHAMP and GRACE altitudes. As DE2 has longer vertical wavelength than DE3, DE2 is expected to penetrate more efficiently into the upper thermosphere than DE3 (Forbes and Garrett, 1979; Forbes et al., 2008). This has been supported by Lühr et al. (2012) (Fig. 5), in studies of ionospheric quantities. They found for DE2 approximately the same relative amplitudes in the EEJ, plasma vertical drift and CTR; while for DE3 the relative amplitude is much more prominent in the EEJ than it is in the other two quantities at CHAMP altitude during the months around August. Since CHAMP and GRACE are at different altitudes, comparing the same tidal amplitudes at two altitudes, may give us some clue of the vertical attenuation of the tides.

During the months around August, DE3 is the most prominent tidal component in the CTR at CHAMP altitude that contributes to WN4, exceeding the amplitude of SPW4 (see Table 3). As expected, at GRACE altitude the amplitudes of all these four tidal components become smaller with different decaying factors. For example, the decay factor, which can be defined as $A_{\mathrm{CHAMP}} / A_{\mathrm{GRACE}}$ ( $A_{\mathrm{CHAMP}}$ and $A_{\mathrm{GRACE}}$ are the tidal amplitudes at the two satellite altitudes respectively), is almost 3 for DE3, and about 2 for SPW4 and SPW2, but the attenuation is even smaller for SW3. Among them the DE3 tide decays so fast that it becomes a secondary tidal component and SPW2 takes the leading role at GRACE altitude. To our surprise, during the months around December solstice at both altitudes the most prominent tide for CTR is neither SPW3 nor DE2, but SW3. The SPW3 and SPW2 reach almost the same amplitude as DE2 at CHAMP altitude, while they exceed the DE2 by far at GRACE altitude. We have looked a little closer into the altitude dependence of the CTR in order to explain the different tidal character with respect to ApexHC. At low (CHAMP) altitude it is mainly the tidal variation of the equatorial trough depletion that governs the DE3 amplitude of CTR. Crest electron densities are less affected by DE3. At GRACE altitude the trough variation is significantly reduced causing the significant amplitude attenuation of DE3 at that altitude. The vertical plasma drift, driving the fountain effect, is determined by the wind dynamo at conjugate foot-prints in the E layer of the related fluxtube.
When talking about vertical plasma velocities at different heights, these map to different latitudes in the E layer. It is well-known that the DE3 tide maximises at the Equator and decays rapidly towards higher latitudes (Forbes et al., 2008; Oberheide et al., 2009). At magnetic latitudes of $\pm 18^{\circ}$, corresponding to an apex height of $800 \mathrm{~km}$, the DE3 intensity seems to be reduced already by a significant amount, and the short vertical wavelength of DE3 also supports the rapid decay. Conversely, the planetary wave SPW4 obviously has a much less height dependence. This is our preferred explanation for the dominance of SPW4 in modulating ApexHC during the months around August.

\subsection{The SW3 tide}

As already reported by Lühr et al. (2012), the SW3 tide is quite prominent in the EEJ, CTR and vertical plasma drift, especially in the last one. The amplitude of the SW3 tide in EEJ is found to be quite large during certain seasons. In particular during fall and winter the SW3 amplitude depends on solar activity (Lühr and Manoj, 2013). In order to deduce the SW3 tidal dependence on solar flux we sorted the CHAMP EIA observations into high (P10.7 $\geq 120 \mathrm{sfu}$ ) and low $(\mathrm{P} 10.7 \leq 120 \mathrm{sfu})$ solar flux bins. The P10.7 represents the mean value of the current day F10.7 and the 81 days averaged F10.7. The DOYs chosen here are between 265 and 030 . Figure 10 presents the local time versus longitudinal distribution of ApexHC during high (left) and low (right) solar flux levels, both the observations (top) and fitted synthetic signals (bottom). The amplitudes and phases of the synthetic tidal components are listed in Table 4. Interestingly, there is little dependence of the two stationary planetary waves on the solar flux level, while the amplitude of SW3 is larger by more than 2.5 during times of elevated solar flux. This strongly suggests that the excitation of SW3, at least during fall and winter, is in favour of a denser ionosphere and thermosphere during times of enhanced EUV fluxes. More research is needed for clarifying the details.

We further sorted the CHAMP ApexHC data by magnetically disturbed $(A p>15)$ and quiet $(A p<15)$ conditions. The amplitudes and phases of the synthetic tidal components are listed in Table 5. Contrary to different solar flux levels, the amplitudes of SW3 show no dependence on magnetic activity. The same behaviour can be stated for SPW3. Different from that, we obtain amplitudes increased by a factor of 2 for the planetary waves SPW2 and DE2 during magnetically quiet periods. This result clearly suggests a wave excitation from below for these two components. During active periods, the upward propagating tidal signal seems to be heavily damped. Consistent with our results, Oberheide et al. (2009) reported an increase of the DE3 amplitude in zonal wind observations at CHAMP altitude from solar maximum to minimum, while at $105 \mathrm{~km}$ altitude the DE3 wind amplitude is independent of the solar cycle phase. 

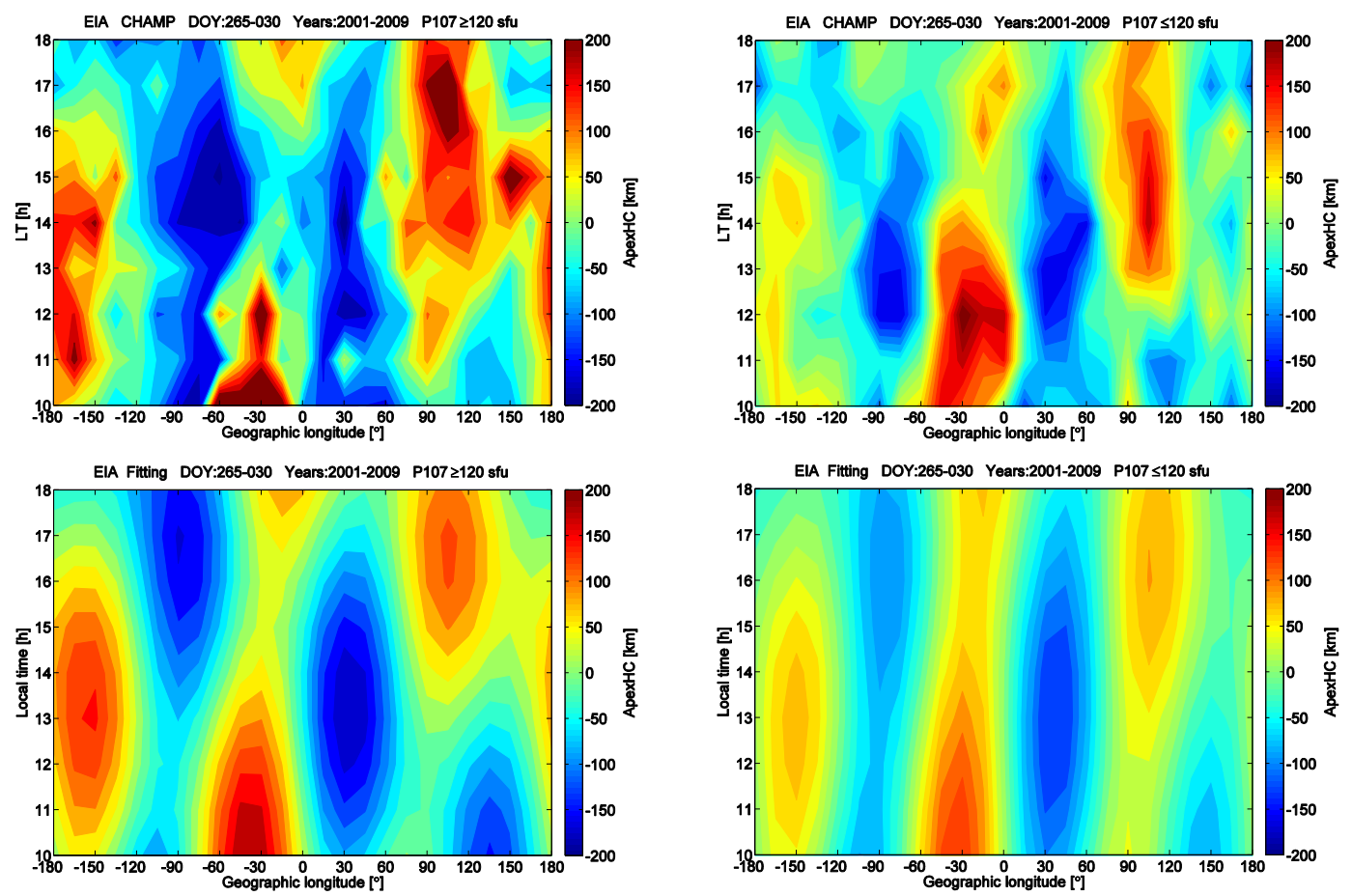

Fig. 10. The local time versus longitudinal distribution of ApexHC during months around December solstice, observations (top) and synthetic signal (bottom) during high (left) and low (right) solar flux levels.

Table 4. Amplitudes and phases of the most prominent tidal components contributing to the modulation of ApexHC during the months around December solstice for different solar flux levels.

\begin{tabular}{cccccc}
\hline \multirow{2}{*}{ Tidal component } & \multicolumn{2}{c}{ P10.7 $\geq 120 \mathrm{sfu}$} & & \multicolumn{2}{c}{ P10.7 $\leq 120 \mathrm{sfu}$} \\
\cline { 2 - 3 } \cline { 5 - 6 } & Amplitude $(\mathrm{km})$ & Phase & & Amplitude $(\mathrm{km})$ & Phase \\
\hline SPW3 & 43.39 & $-37^{\circ}$ & & 44.90 & $-31^{\circ}$ \\
SPW2 & 36.98 & $-25^{\circ}$ & & 33.39 & $-33^{\circ}$ \\
DE2 & 48.57 & $17.4 \mathrm{~h}$ & & 37.61 & $16.2 \mathrm{~h}$ \\
SW3 & 75.92 & $19.3 \mathrm{~h}$ & & 29.57 & $19.4 \mathrm{~h}$ \\
\hline
\end{tabular}

\subsection{The EIA hemispheric asymmetry}

So far the asymmetry of the EIA has never been discussed in terms of tides. We have obtained rather distinct longitudinal patterns for the EIA asymmetry. Around June solstice the ionization anomaly is displaced by several degrees in latitude towards north in the western sector from $-180^{\circ} \mathrm{E}$ to $-60^{\circ} \mathrm{E}$ in longitude, while in the remaining part the EIA is slightly displaced southward. This asymmetry can well be described by the planetary waves, SPW1 and SPW2. Although we cannot offer a conclusive explanation for the observed EIA displacement, it is worth noting that Lühr and Manoj (2013) report about an enhanced EEJ intensity at all daytime hours in the same western longitude sector during June solstice months (see their Fig. 18). This suggests thermospheric winds as the reason for the northward shift of the EIA.
During December solstice months we find a similar longitudinal pattern for the asymmetry, but this time the sector for northward displacement is shifting eastward with local time. Now the tidal component D0 is dominating the spectrum. When taking the phase into account D0 causes the EIA to be displaced worldwide furthest north at 00:00 UT, then the EIA moves everywhere southward and reaches the lowest position at 12:00 UT. After that it returns to the north again. Since the EIA does not exist all around the globe this diurnal motion is only observable in the sunlit part of the world where the anomaly is developed. From our results we can deduce that the motion is clearest in the Western Hemisphere. The most probable cause for the diurnal oscillation of the EIA asymmetry is an interaction of the migrating tide with the latitude variation of the magnetic equator. It is not clear why this interaction takes place around December but not around June. 
Table 5. Amplitudes and phases for the most prominent tidal components contributing to the modulation of ApexHC during the months around December solstice for magnetic quiet and disturbance conditions.

\begin{tabular}{cccccc}
\hline \multirow{2}{*}{ Tidal component } & \multicolumn{2}{c}{ Ap $>15$} & & \multicolumn{2}{c}{ Ap $<15$} \\
\cline { 2 - 3 } \cline { 5 - 6 } & Amplitude $(\mathrm{km})$ & Phase & & Amplitude $(\mathrm{km})$ & Phase \\
\hline SPW3 & 49.92 & $-33^{\circ}$ & & 47.34 & $-33^{\circ}$ \\
SPW2 & 18.40 & $-26^{\circ}$ & & 35.82 & $-32^{\circ}$ \\
DE2 & 22.49 & $15.3 \mathrm{~h}$ & & 41.96 & $16.1 \mathrm{~h}$ \\
SW3 & 39.83 & $19.5 \mathrm{~h}$ & & 41.06 & $19.6 \mathrm{~h}$ \\
\hline
\end{tabular}

\section{Conclusion}

Based on nearly nine years of observations from both CHAMP and GRACE the tidal signatures of the EIA have been analysed in this study. The main findings can be summarised as:

1. The EIA shows longitudinal WN4 and WN3 patterns during the months around August and December solstice, respectively, both in CTR and ApexHC from CHAMP and GRACE observations.

2. For CTR, the leading tidal component contributing to WN4 is DE3, while for WN3 the stationary planetary wave SPW3 is as important as DE2. For ApexHC the stationary planetary waves SPW4/SPW3 exceed the DE3/DE2 amplitudes, taking the leading role in contributing to the longitudinal WN4/WN3 patterns. During the months around August the DE3 tide in ApexHC is really weak compared to SPW4.

3. The dominance of DE3 in ionospheric electrodynamics seems to be confined to equatorial latitudes and moderate heights, while SPW4 is less damped with increasing altitude. SPW4 is assumed to be caused by an interaction of DW1 with DE3 at E region heights.

4. During the months around December solstice the SW3 tide is prominent both in CTR and ApexHC. Furthermore, SW3 shows a strong dependence on solar flux, thus favouring a denser ionosphere and thermosphere while it hardly depends on magnetic activity.

5. The inter-hemispheric asymmetry of the EIA shows only WN1 and WN2 longitudinal structures with northward displacements in the $-180^{\circ} \mathrm{E}$ to $-60^{\circ} \mathrm{E}$ longitude sector and southward displacement in the rest. The dominating WN1 is stationary during June solstice, while it shows an eastward tilted LT evolution during December solstice, which can be described by the D0 tidal component.

Further studies are needed for explaining the causes of the various tidal components modulating the equatorial ionization anomaly.

\section{Appendix A}

\section{List of abbreviations}

$\begin{array}{ll}\text { ACL: } & \text { Asymmetry of Crest Latitude } \\ \text { ApexHC: } & \text { Apex Height of Crest } \\ \text { CHAMP: } & \text { CHAllenging Minisatellite Payload } \\ \text { COSMIC: } & \text { Constellation Observing System of } \\ & \text { Meteorology, Ionosphere, and Cli- } \\ & \text { mate } \\ \text { CTR: } & \text { Crest-to-Trough Ratio } \\ \text { DOY: } & \text { Days of Year } \\ \text { EEJ: } & \text { Equatorial Electrojet } \\ \text { EIA: } & \text { Equatorial Ionization Anomaly } \\ \text { GAIA: } & \text { Ground-to-topside model of Atmo- } \\ & \text { sphere and Ionosphere for Aeron- } \\ \text { GRACE: } & \text { omy } \\ & \text { Gravity Recovery And Climate Ex- } \\ \text { KBR: } & \text { Keriment } \\ \text { LT: } & \text { Locand Time } \\ \text { MCL: } & \text { Mean Crest Latitude } \\ \text { MLT: } & \text { Mesosphere-lower Thermosphere } \\ \text { PLP: } & \text { Planar Langmuir Probe } \\ \text { TEC: } & \text { Total Electron Content } \\ \text { TIMED/GUVI: } & \text { Thermosphere, Ionosphere, Meso- } \\ & \text { sphere Energetics and Dynam- } \\ & \text { ics/Global Ultraviolet Imager } \\ \text { TIME-GCM: } & \text { Thermosphere Ionosphere Meso- } \\ & \text { sphere Electrodynamics General } \\ & \text { Circulation Model } \\ \text { UT: } & \text { Universal Time } \\ \text { WN4: } & \text { Wavenumber 4, similar as WN1, } \\ & \text { WN2, WN3 }\end{array}$

Acknowledgements. The CHAMP and GRACE missions were sponsored by the Space Agency of the German Aerospace Centre (DLR) through funds of the Federal Ministry of Economics and Technology. This study is supported by the Doctoral Fund of Ministry of Education of China (Grant No. 200804860012) and the Ocean Public Welfare Scientific Research Project, SOA of China (201005017). The work of C. Xiong is supported by the Alexander von Humboldt foundation through a Research Fellowship for Postdoctoral Researchers. 
The service charges for this open access publication

have been covered by a Research Centre of the

Helmholtz Association.

Topical Editor C. Jacobi thanks E. de Paula and one anonymous referee for their help in evaluating this paper.

\section{References}

Duncan, R. A.: The equatorial F-region of the ionosphere, J. Atmos. Terr. Phys., 18, 89-100, 1959.

England, S. L.: A review of the effects of non-migrating atmospheric tides on the earth's low-latitude ionosphere, Space Sci. Rev., 168, 211-236, doi:10.1007/s11214-011-9842-4, 2011.

England, S. L., Maus, S., Immel, T. L., and Mende, S. B.: Longitude variation of the E-region electric fields caused by atmospheric tides, Geophys. Res. Lett., 33, L21105, doi:10.1029/2006GL027465, 2006.

England, S. L., Immel, T. J., Huba, J. D., Hagan, M. E., Maute, A., and DeMajistre, R.: Modeling of multiple effects of atmospheric tides on the ionosphere: An examination of possible coupling mechanisms responsible for the longitudinal structure of the equatorial ionosphere, J. Geophys. Res., 115, A05308, doi:10.1029/2009JA014894, 2010.

Forbes, J. M. and Garrett, H. B.: Theoretical studies of atmospheric tides, Rev. Geophys. Space Phys., 17, 1951-1981, 1979.

Forbes, J. M., Russell, J., Miyahara, S., Zhang, X., Palo, S., Mlynczak, M., Mertens, C. J., and Hagan, M. E.: Tropospherethermosphre tidal coupling as measured by the SABER instrument on TIMED during July-September 2002, J. Geophys. Res., 111, A10S06, doi:10.1029/2005JA011492, 2006.

Forbes, J. M., Zhang, X., Palo, S., Russell, J., Mertens, C. J., and Mlynczak, M.: Tidal variability in the ionospheric dynamo region, J. Geophys. Res., 113, A02310, doi:10.1029/2007JA012737, 2008.

Forbes, J. M., Zhang, X., and Bruinsma, S.: Middle and upper thermosphere density structures due to nonmigrating tides, J. Geophys. Res., 117, A11306, doi:10.1029/2012JA018087, 2012.

Hagan, M. E. and Forbes, J. M.: Migrating and nonmigrating diurnal tides in the middle and upper atmosphere excited by tropospheric latent heat release, J. Geophys. Res., 107, 4754, doi:10.1029/2001JD001236, 2002.

Hagan, M. E. and Forbes, J. M.: Migrating and nonmigrating semidiurnal tides in the upper atmosphere excited by tropospheric latent heat release, J. Geophys. Res., 108, 1062, doi:10.1029/2002JA009466, 2003.

Hagan, M. E., Maute, A., and Roble, R. G.: Tropospheric tidal effects on the middle and upper atmosphere, J. Geophys. Res., 114, A01302, doi:10.1029/2008JA013637, 2009.

Häusler, K. and Lühr, H.: Nonmigrating tidal signals in the upper thermospheric zonal wind at equatorial latitudes as observed by CHAMP, Ann. Geophys., 27, 2643-2652, doi:10.5194/angeo27-2643-2009, 2009.

He, M., Liu, L., Wan, W., Lei, J., and Zhao, B.: Longitudinal modulation of the $\mathrm{O} / \mathrm{N}_{2}$ column density retrieved from TIMED/GUVI measurement, Geophys. Res. Lett., 37, L20108, doi:10.1029/2010GL045105, 2010.

Immel, T. J., Sagawa, E., England, S. L., Henderson, S. B., Hagan, M. E., Mende, S. B., Frey, H. U., Swenson, C. M., and Paxton, L. J.: Control of equatorial ionospheric morphol- ogy by atmospheric tides, Geophys. Res. Lett., 33, L15108, doi:10.1029/2006GL026161, 2006.

Kil, H., Oh, S.-J., Kelley, M. C., Paxton, L. J., England, S. L., Talaat, E., Min, K.-W., and Su, S. Y.: Longitudinal structure of the vertical $\boldsymbol{E} \times \boldsymbol{B}$ drift and ion density seen from ROCSAT-1, Geophys. Res. Lett., 34, L14110, doi:10.1029/2007GL030018, 2007.

Kil, H., Paxton, L. J., Lee, W. K., Ren, Z., Oh, S.-J., and Kwak, Y.-S.: Is DE2 the source of the ionospheric wavenumber 3 longitudinal structure?, J. Geophys. Res., 115, A11319, doi:10.1029/2010JA015979, 2010.

Lühr, H. and Manoj, C. N.: The complete spectrum of the equatorial electrojet related to solar tides: CHAMP observations, Ann. Geophys., in review, 2013.

Lühr, H. and Xiong, C.: IRI-2007 model overestimates electron density during the 23/24 solar minimum, Geophys. Res. Lett., 37, L23101, doi:10.1029/2010GL045430, 2010.

Lühr, H., Rother, M., Häusler, K., Alken, P., and Maus, S.: The influence of non-migrating tides on the longitudinal variation of the equatorial electrojet, Geophys. Res., 113, A08313, doi:10.1029/2008JA013064, 2008.

Lühr, H., Rother, M., Häusler, K., Fejer, B., and Alken, P.: Direct comparison of non-migrating tidal signatures in the electrojet, vertical plasma drift and equatorial ionization anomaly, J. Atmos. Solar-Terr. Phys., 75-76, 31-43, doi:10.1016/j.jastp.2011.07.009, 2012.

McNamara, L., Cooke, D. L., Valladares, C. E., and Reinisch, B. W.: Comparison of CHAMP and Digisonde plasma frequencies at Jicamarca. Peru, Radio Sci., 42, RS2005, doi:10.1029/2006RS003491, 2007.

Oberheide, J., Forbes, J. M., Hausler, K., Wu, Q., and Bruinsma, S. L.: Tropospheric tides from 80 to $400 \mathrm{~km}$ : propagation, interannual variability, and solar cycle effects, J. Geophys. Res., 114, D00I05, doi:10.1029/2009JD012388, 2009.

Oberheide, J., Forbes, J. M., Zhang, X., and Bruinsma, S. L.: Wave-driven variability in the ionosphere-thermospheremesosphere system from TIMED observations: What contributes to the "wave 4"?, J. Geophys. Res., 116, A01306, doi:10.1029/2010JA015911, 2011.

Pancheva, D. and Mukhtarov, P.: Global response of the ionosphere to atmospheric tides forced from below: Recent progress based on satellite measurements, Space Sci. Rev., 161, 1-35, doi:10.1007/s11214-011-9837-1, 2011.

Pancheva, D., Miyoshi, Y., Mukhtarov, P., Jin, H., Shinagawa, H., and Fujiwara, H.: Global response of the ionosphere to atmospheric tides forced from below: Comparison between COSMIC measurements and simulations by atmosphereionosphere coupled model GAIA, J. Geophys. Res., 117, A07319, doi:10.1029/2011JA017452, 2012.

Reigber, C., Lühr, H., and Schwintzer, P.: CHAMP mission status, Adv. Space Res., 30, 129-134, 2002.

Rishbeth, H.: The equatorial F-layer: progress and puzzles, Ann. Geophys., 18, 730-739, doi:10.1007/s00585-000-0730-6, 2000.

Sagawa, E., Immel, T. J., Frey, H. U., and Mende, S. B.: Longitudinal structure of the equatorial anomaly in the nighttime ionosphere observed by IMAGE/FUV, J. Geophys. Res., 110, A11302, doi:10.1029/2004JA010848, 2005.

Scherliess, L., Thompson, D. C., and Schunk, R. W.: Longitudinal variability of low- latitude total electron content: Tidal influences, J. Geophys. Res., 113, A01311, 
doi:10.1029/2007JA012480, 2008.

Tapley, B. D., Bettadpur, S., Watkins, M., and Reigber, C.: The gravity recovery and climate experiment: Mission overview and early results, Geophys. Res. Lett., 31, L09607, doi:10.1029/2004GL019920, 2004.

Wan, W., Xiong, J., Ren, Z., Liu, L., Zhang, M.-L., Ding, F., Ning, B., Zhao, B., and Yue, X.: Correlation between the ionospheric WN4 signature and the upper atmospheric DE3 tide, J. Geophys. Res., 115, A11303, doi:10.1029/2010JA015527, 2010.

Xiong, C., Park, J., Lühr, H., Stolle, C., and Ma, S. Y.: Comparing plasma bubble occurrence rates at CHAMP and GRACE altitudes during high and low solar activity, Ann. Geophys., 28, 1647-1658, doi:10.5194/angeo-28-1647-2010, 2010.
Xiong, C., Lühr, H., and Ma, S. Y.: The magnitude and interhemispheric asymmetry of equatorial ionization anomaly-based on CHAMP and GRACE observations, J. Atmos. Solar-Terr. Phys., in review, 2013.

Zhang, Y., England, S., and Paxton, L. J.: Thermospheric composition variations due to nonmigrating tides and their effect on ionosphere, Geophys. Res. Lett., 37, L17103, doi:10.1029/2010GL044313, 2010. 